\title{
CRIANÇAS QUE UTILIZAM OS SERVIÇOS DE SAÚDE MENTAL: CARACTERIZAÇÃO DA POPULAÇÃO EM UMA CIDADE DO SUL DO BRASIL
}

\section{CHILDREN WHO USE MENTAL HEALTH SERVICES: CHARACTERIZATION OF THE POPULATION IN A CITY IN SOUTHERN BRAZIL}

\author{
Josiane da Silva Delvan ${ }^{1}$ \\ João Rodrigo Maciel Portes ${ }^{2}$ \\ Maiara Pereira Cunha ${ }^{3}$ \\ Marina Menezes ${ }^{4}$ \\ Eduardo José Legal ${ }^{5}$
}

Delvan, JS et al. Crianças que utilizam os serviços de saúde mental: caracterização da população em uma cidade do sul do Brasil. Rev Bras Cresc e Desenv Hum 2010; 20(2): 228-237

\section{Resumo:}

Estudos têm indicado que a população infantil constitui grande demanda para os serviços de saúde mental. Neste trabalho, objetivou-se caracterizar o perfil da população infantil que procurou o atendimento nos serviços de saúde mental, na cidade de Itajaí entre os anos de 2002 a 2007. Os dados desta pesquisa foram levantados junto aos prontuários da população atendida em dois serviços públicos de saúde mental existentes na cidade, caracterizando este estudo como documental, exploratório e descritivo. Os resultados encontrados indicam que os transtornos mentais mais relatados como queixa são o Transtorno de Déficit de Atenção e Comportamento Disruptivo totalizando 28,7\%, Transtornos de Ansiedade 9,6\% e os Transtornos de Aprendizagem 7,8\% desta população que procura atendimento nos serviços de saúde mental. Em relação aos Problemas de Comportamento encontram-se os Problemas nas Relações 13,3\%, Condições Adicionais que Podem ser Foco de Atenção Clínica 2,7\% e Agressividade 2,1\%. Com base nestes dados, deve-se repensar o planejamento de políticas públicas para que haja a promoção e, sobretudo, a prevenção dos transtornos mentais que acometem a população infantil.

Palavras-chave: criança; saúde mental; transtornos mentais.

1 Doutora. Professora de Psicologia do Desenvolvimento Humano do curso de Psicologia da Universidade do Vale do Itajaí UNIVALI

2 Acadêmico do curso de Psicologia da UNIVALI. Bolsista de Iniciação Científica. joaorodrigo@univali.br

3 Acadêmica do curso de Psicologia da UNIVALI. maiara_pereira@yahoo.com.br

4 Mestre. Professora de Psicopatologia Infantil do Curso de Psicologia da Universidade do Vale do Itajaí - UNIVALI. menezesmarina@univali.br

5 Doutor. Professor de Psicopatologia Geral do curso de Psicologia da Universidade da Vale do Itajaí - UNIVALI. edulegal@univali.br

Universidade do Vale do Itajaí. Curso de Psicologia, bloco 25b, sala 401. Rua: Uruguai, 458. Centro. Itajaí, SC. CEP $88302-$ 202 josidelvan@univali.br.

Financiamento: UNIVALI/ Governo do Estado de Santa Catarina 


\begin{abstract}
:
The literature has indicated that the children population represents the majority of people who use mental health services. In this study, the objective is to characterize the profile of the children population who has looked for a mental health service in the town of Itajaí from 2002 to 2007. Data were collected from the documents of this population who attended the two mental health services.This study is a documentary, exploratory and descriptive one. The most frequent mental problens (28, 7\%) of the cases were. Déficit of Attention and the Disruptive Behaviour, in this category the Deficit of Attention/hyperactivity, was the most frequent. The problem about behaviour, $(13,3 \%)$ of data, are the relationship problems. Based on these data, it is extremely urgent to analyse and rethink Public Social Policies in the prevention of mental problems which affect the children population.
\end{abstract}

Key words: child; mental health; mental bewilderment.

\section{INTRODUÇÃO}

Com o desenvolvimento tecnológico e a mobilidade geográfica que marca este século, o discurso científico médico-psicológico tornou-se o referencial para as práticas destinadas ao cuidado de crianças e adolescentes e passou a orientar a relação pais-filhos. Os cuidados dirigidos a esta população passaram a ser foco de maior interesse de profissionais da saúde e se tornaram também uma regra social a partir do momento em que determinadas leis foram criadas para garantir seus direitos na Constituição Federal de 1988 e no Estatuto da Criança e do Adolescente em 1990. Alguns destes direitos se referem à educação e à saúde que devem ser garantidos pelos pais ou responsáveis e pelo Estado, através dos serviços públicos; como o serviço de saúde mental. Consoante a Reforma Psiquiátrica, a saúde mental vem há mais de vinte anos desenvolvendo-se no Brasil, de forma tímida, porém, com eficientes iniciativas do poder público em relação às crianças e adolescentes com transtornos mentais.

O conceito de saúde mental, que difere do modelo biomédico considera o ser humano inserido num contexto social cujas implicações estão intimamente relacionadas à fase do de- senvolvimento que vivencia. Nesse sentido, há que se considerar que a saúde mental se refere a um equilíbrio dinâmico que resulta da capacidade da pessoa de pensar e adaptarse à realidade, de sua capacidade de desempenhar funções sociais, e também de sua capacidade em lidar com a maior parte dos problemas da vida cotidiana. Considerando estas idéias, a prevenção e a reabilitação das doenças mentais são partes integrantes do domínio da saúde mental ${ }^{1}$.

No Brasil, o Ministério da Saúde iniciou ações no nível da Atenção Básica, definindo o programa de saúde mental contemplando o que a Psicologia propõe para o atendimento de crianças e adolescentes com transtornos mentais graves, através da implantação dos Centros de Atenção Psicossocial para Infância e Adolescência - CAPSi, que surgiram com a Portaria MS 336/02 a qual modificou essa configuração, prevendo-se um modelo com orientações específicas ao planejamento assistencial em saúde mental infantojuvenil ${ }^{2}$.

A reformulação da assistência pública nesse setor traz uma nova prática clínica, demarcando uma proposta que articula a subjetividade e a dimensão política na organização da assistência. Os serviços em saúde mental foram organizados em torno de uma visão huma- 
nitária de tratamento e de inserção social aos sofredores psíquicos, substituindo e superando a idéia de manicômios nos quais o ser humano era excluído da sociedade ${ }^{3}$.

De acordo com o relatório da Organização Mundial de Saúde (OMS) de 2005, 30\% dos países ainda não possuem políticas de saúde mental para a sua população e, mais grave, $90 \%$ não desenvolveram políticas de saúde mental que incluam crianças e adolescentes ${ }^{4}$.

Levantamentos desta natureza são datados da década de 80 , seguidos por outros realizados principalmente em clínicas escola que dispõem do Serviço de Psicologia ${ }^{5-13}$.

Atualmente, os serviços de saúde mental para o atendimento da população infantil disponíveis em Itajaí caracterizam-se por um serviço público de saúde mental e uma clínica escola de Psicologia. A coleta dos dados foi autorizada pelos dois serviços de saúde mental, mediante parecer favorável do Comitê de Ética em Pesquisa e ocorreu durante os meses de junho e julho de 2007.

Dessa maneira, o presente trabalho visa a descrever o perfil das crianças e adolescentes atendidos nos serviços de saúde mental na cidade de Itajaí com objetivo de identificar os problemas emocionais e comportamentais mais freqüentes desta clientela que motivaram a procura por tais serviços.

\section{MÉTODO}

Trata-se de uma pesquisa de abordagem quantitativa, definindo-se como documental, exploratória e descritiva. A partir da elaboração da ficha para a coleta dos dados nos prontuários, levantaram-se as informações para a caracterização da população atendida nos serviços de saúde mental disponíveis na cidade de Itajaí, SC. Esta é privilegiada por sua localização estratégica: $100 \mathrm{~km}$ ao norte de Joinville e ao sul, Florianópolis, Blumenau e Brusque localizam-se a 50 km. Itajaí está loca- lizada no litoral catarinense com população estimada em torno de 170.000 habitantes.

Neste estudo tomou-se como base a definição da Organização Mundial de Saúde que também foi adotada pelo Ministério da Saúde, segundo a qual o período da infância vai até os nove anos de idade e a partir dos dez anos já se inicia a fase da adolescência ${ }^{14}$.

A amostra total ficou composta por 564 prontuários. Os dados foram coletados a partir dos prontuários dos pacientes com idade de três a dez anos, de ambos os sexos, nas duas instituições da cidade que oferecem o serviço de saúde mental para crianças e adolescentes. Foi elaborada uma ficha para sistematização dos dados de identificação da população foco do estudo: idade, sexo, escolaridade, com quem reside, número de filhos na família e posição ocupada pela criança na prole; a fonte do encaminhamento para os serviços de saúde mental e as queixas desta população que motivaram a procura dos serviços. Para a realização deste levantamento, foram coletados, além dos casos registrados de janeiro de 2002 a dezembro de 2006, também os dados referentes aos casos iniciados no período de janeiro até junho de 2007.

Os dados coletados foram tabulados e calculadas as respectivas percentagens. Para as diferenças entre sexo e idade foi aplicada a análise de variância para dados nãoparamétricos através do SPSS 10.0 para Windows.

\section{RESULTADOS E DISCUSSÃO}

Inicialmente na Tabela 1 são apresentados os dados referentes ao período de tempo pesquisado, sendo que no ano de 2002 obteve-se uma incidência de $16,7 \%$ de atendimentos a crianças e adolescentes. Desde então, pode-se observar que há uma crescente procura da população pelos serviços de saúde mental disponíveis na cidade conforme indi- 
cado na tabela desde o ano de 2003 (com $13,7 \%)$, seguido do ano de 2004 (18,3\%), 2005 (19,1\%) e 2006 (20,6\%). Em 2007 foi pesquisado apenas o primeiro semestre com $10,6 \%$. Salienta-se que em $1,1 \%$ dos prontuá- rios não foram constatados dados referentes ao ano de atendimento.

Na Tabela 1 pode-se observar a distribuição da amostra por sexo. Verifica-se um predomínio de meninos (66\%) seguido por 33,2\%

Tabela 1: Distribuição da amostra da população atendida nos serviços de saúde mental referente aos anos pesquisados, quanto ao sexo, a idade e a escolaridade da criança e ou adolescente.

\begin{tabular}{|c|c|c|c|}
\hline Dados pessoais & Variáveis & Frequência & Porcentagem (\%) \\
\hline Ano de atendimento & $\begin{array}{l}2002 \\
2003 \\
2004 \\
2005 \\
2006 \\
2007\end{array}$ & $\begin{array}{c}94 \\
77 \\
103 \\
108 \\
116 \\
60\end{array}$ & $\begin{array}{l}16,7 \% \\
13,7 \% \\
18,3 \% \\
19,1 \% \\
20,6 \% \\
10,6 \%\end{array}$ \\
\hline $\begin{array}{l}\text { Sexo } \\
\text { Idade } \\
\end{array}$ & $\begin{array}{l}\text { Masculino } \\
\text { Feminino } \\
3 \\
4 \\
5 \\
6 \\
7 \\
8 \\
9 \\
10\end{array}$ & $\begin{array}{c}372 \\
187 \\
30 \\
42 \\
53 \\
54 \\
92 \\
102 \\
97 \\
94\end{array}$ & $\begin{array}{c}66 \% \\
33,2 \% \\
5,3 \% \\
7,4 \% \\
9,4 \% \\
9,6 \% \\
16,3 \% \\
18,1 \% \\
17,2 \% \\
16,7 \%\end{array}$ \\
\hline Escolaridade & 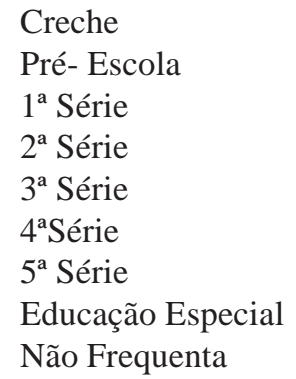 & $\begin{array}{c}79 \\
49 \\
99 \\
128 \\
71 \\
66 \\
19 \\
5 \\
17\end{array}$ & $\begin{array}{c}14 \% \\
8,7 \% \\
17,6 \% \\
22,7 \% \\
12,6 \% \\
11,7 \% \\
3,4 \% \\
0,9 \% \\
3,0 \%\end{array}$ \\
\hline
\end{tabular}

de meninas, sendo que em $0,9 \%$ dos prontuários não foram encontradas informações a respeito do sexo dos pacientes. Outros estudos corroboram com os dados apresentados, demonstrando a primazia do sexo masculino nos atendimentos em serviços de saúde mental disponíveis 3,4,6,7,12,15.
Ainda na Tabela 1 apresenta-se a distribuição da amostra em função da idade das crianças e adolescentes, sendo calculadas a partir da data de nascimento e com base na data de inscrição, considerando os anos completos. Observa-se um predomínio da faixa etária de crianças e adolescentes no início da fase esco- 
lar, de sete a dez anos, dado também apontado em outro estudo na década de $90^{6}$.

Todavia, em outros estudos constata-se certa variação em relação à faixa etáriaa,7,12,15. Verifica-se no presente gráfico que a maior incidência de crianças encaminhadas para o atendimento em saúde mental ocorreu na idade de oito anos $(18,1 \%)$, seguida de crianças com nove anos $(17,2 \%)$ adolescentes dez anos $(16,7 \%)$ e crianças com sete anos (16,3\%).

De acordo com a Tabela 1 têm-se os dados relativos à distribuição da população estudada quanto ao nível de escolaridade. Nota-se que há uma incidência maior das crianças na $2^{\mathrm{a}}$ série (22,7\%), em seguida crianças oriundas da $1^{a}$ série $(17,6 \%)$ e crianças de creche $(14 \%)$. Lembrando que no item creche constam crianças com idade abaixo de três anos. Em 5,5\% dos prontuários não foram constatadas informações sobre o nível de escolaridade. O predomínio das crian- ças que estudam na $1^{\mathrm{a}}$ e $2^{\mathrm{a}}$ séries também foi demonstrado por outros autores ${ }^{4,7}$.

A Tabela 2 apresenta dados referentes à composição familiar. Segundo os prontuários pesquisados de 2002 a 2007 no Centro de Atenção Psicossocial para Infância e Adolescência (CAPsi) e na Clínica Escola de Psicologia, há uma prevalência de crianças e ou adolescentes procedentes de família nuclear (51,7\%), seguida por crianças e ou adolescentes em que a mãe é o chefe da família (29,6\%), seguido dos avós (5,6\%) como os responsáveis, do pai (4,3\%) como o chefe da família, tios $(2,0 \%)$ e outros $(1,4 \%)$. Em $5,4 \%$ dos prontuários não constavam informações sobre a composição familiar. Consoante o estudo realizado numa clínica-escola a maioria das crianças pesquisadas também moram com os pais (família nuclear), sendo que os demais contam com a presença apenas da mãe, e há ainda aqueles que residem exclusivamente com o pai ${ }^{6}$.

Tabela 2: Distribuição da amostra da população atendida nos serviços de saúde mental no período de 2002 a 2007 em função da sua composição familiar, o números de filhos da família e a posição que a criança e ou adolescente ocupa na prole.

\begin{tabular}{llcc}
\hline Dados pessoais & Variáveis & Freqüência & Porcentagem (\%) \\
\hline Composição & Família Nuclear & 288 & $51,7 \%$ \\
Familiar & Mãe & 165 & $29,6 \%$ \\
& Pai & 24 & $4,3 \%$ \\
& Avós & 31 & $5,6 \%$ \\
& Tios & 11 & $2,0 \%$ \\
& Outros & 8 & $1,4 \%$ \\
\hline Número de Filhos & 1 & 127 & $22,5 \%$ \\
da Família & 2 & 214 & $37,9 \%$ \\
& 3 & 94 & $16,7 \%$ \\
& 4 & 43 & $7,6 \%$ \\
& 5 & 11 & $2,0 \%$ \\
& 6 & 7 & $1,2 \%$ \\
& 7 & 3 & $0,5 \%$ \\
& 8 & 3 & $0,5 \%$ \\
& 9 & 2 & $0,4 \%$ \\
Posição ocupada & 10 & 1 & $0,2 \%$ \\
pela criança na & Gêmeos & 2 & $0,4 \%$ \\
prole & $1^{\text {a }}$ & 261 & $46,3 \%$ \\
& $2^{\text {a }}$ & 145 & $25,7 \%$ \\
& $3^{a}$ & 45 & $8,0 \%$ \\
& $4^{\text {a }}$ & 14 & $2,5 \%$ \\
& $5^{\text {a }}$ & 7 & $1,2 \%$ \\
& $6^{\text {a }}$ & 4 & $0,7 \%$ \\
& $7^{\text {a }}$ & 5 & $0,9 \%$ \\
& $8^{\text {aa }}$ & 1 & $0,2 \%$ \\
\hline
\end{tabular}


Na distribuição da amostra em função da composição familiar, a Tabela 2 apresenta a quantidade de filhos. Nas famílias das crianças e adolescentes atendidas nos serviços de saúde mental, foi encontrada uma maior concentração de famílias com dois filhos (37,9\%) seguidas por famílias com filho único (22,5\%) e três filhos (16,7\%), sendo que em 10,5\% dos prontuáruis não foram encontradas informações sobre o número de filhos da família das crianças e adolescentes atendidos. Outro estudo também aponta uma alta incidência de famílias com dois filhos ${ }^{6}$.

Os dados referentes à posição ocupada pela criança e ou adolescente na prole são apresentados na Tabela 2, tendo uma concentração maior dos primogênitos $(46,3)$, seguida do segundo filho $(25,7 \%)$ e terceiro filho $(8,0 \%)$, sendo que em $14,2 \%$ dos casos não foram constatadas informações sobre a posição ocupada na prole. Os dados referentes ao estudo realizado em uma clínica-escola em São Paulo são bem semelhantes, pois a maior incidência também foi observada com os primogênitos seguidos do segundo, terceiro e quarto filho ${ }^{6}$.

Na Tabela 3 constam os dados referentes às fontes de encaminhamento para os Serviços de Saúde Mental na cidade de Itajaí, SC. A maior incidência foi de busca espontânea do serviço (25\%), seguida de encaminhamentos das crianças e ou adolescentes feitas pelas escolas (16\%), encaminhamentos dos médicos para o serviço de saúde mental (14,2\%), sendo que em $18,4 \%$ dos casos não constavam informações sobre a fonte de encaminhamento da criança e ou adolescente. Estes dados, quando comparados a outros estudos, mostram-se divergentes, pois nessas investigações os encaminhamentos são feitos em maior quantidade pelos profissionais da saúde, seguidas das instituições escolares e pela iniciativa dos familiares ${ }^{6,7}$. Per-

Tabela 3: Distribuição das fontes de encaminhamento da população aos serviços de saúde mental neste período.

\begin{tabular}{lcc}
\hline Fonte de Encaminhamento & Freqüência & $\mathbf{( \% )}$ \\
\hline Busca Espontânea & 141 & 25 \\
Escolas & 96 & 17,1 \\
Médico & 80 & 14,2 \\
Unidade Básica de Saúde & 60 & 10,6 \\
Conselho Tutelar & 23 & 4,1 \\
Ambulatório de Medicina Familiar da UNIVALI** & 11 & 2,0 \\
Psicólogo & 10 & 1,8 \\
Clínica Escola de Fonoaudiologia UNIVALI** & 7 & 1,2 \\
CAPSi (Centro de Atenção Psicossocial para Infância e Adolescência) & 5 & 0,9 \\
Secretaria Municipal de Saúde & 5 & 0,9 \\
Abrigos & 4 & 0,7 \\
Secretaria Municipal de Educação & 3 & 0,5 \\
APAE (Associação de Pais e Amigos dos Excepcionais) & 2 & 0,4 \\
Clínica Escola de Fisioterapia da Univali & 2 & 0,4 \\
Fonoaudiólogo & 2 & 0,4 \\
Outros** & 7 & 1,4 \\
Prontuário sem essa informação & 106 & 18,4 \\
\hline Total & & 100,0 \\
\hline
\end{tabular}

**Outros : Fórum, Cendi (Centro de Desenvolvimento Integral), Saps (Serviço de Apoio ao Portador de Surdez), Rede de programa de pesquisa de Itajaí, Padef (Programa de Atenção aos Discentes, Egressos e Funcionários da UNIVALI), Hospital, Cemesp (Centro Municipal de Educação Alternativa de Itajaí). *** UNIVALI: esta fonte constou apenas dos levantamentos realizados nos prontuários da Clínica Escola de Psicologia. 
cebe-se, então, uma grande variação entre os dados das pesquisas quando comparados em relação à fonte de encaminhamento, visto que a diferença é constatada principalmente na categoria "procura espontânea", que não está presente e não é classificada nos demais estudos. Infere-se que tal possa ser um indicativo de que alguns pais atribuem à criança e ou adolescente algum problema, e desta forma procuram ajuda nos serviços de saúde.

Neste estudo, optou-se em separar os dados referentes aos diagnósticos clínicos - transtornos mentais em uma categoria e os problemas comportamentais em outra, devido à classificação existente no DSM-IV-TR ${ }^{16}$. De acordo com esta literatura, a descrição dos transtornos mentais é mais detalhada quanto à etiologia, sintomatologia, epidemiologia e prognóstico. Já os problemas comportamentais são tratados de maneira menos descritiva e considerados menos graves que os transtornos mentais.

A Tabela 4 apresenta dados referentes aos três diagnósticos clínicos com maior incidência encontrados nos prontuários, e categorizados conforme o DSM-IV-TR. Através do levantamento de dados constatou-se a presença de di- agnósticos de 21 transtornos mentais diferentes, destacando-se com uma maior prevalência os transtornos de déficit de atenção e de comportamento disruptivos com (28,7\%). Nessa categoria estão incluídos transtornos como transtorno de déficit de atenção/hiperatividade, transtorno de conduta, transtorno desafiador de oposição, transtorno de comportamento disruptivo sem outra especificação. Em seguida, com maior incidência, aparecem os transtornos de ansiedade com $(9,6 \%)$ caracterizados pelos transtornos de ataque de pânico, agorafobia, transtorno de pânico, agorafobia sem histórico de transtorno de pânico, fobia específica, fobia social, transtorno obsessivo-compulsivo, transtorno de estresse pós-traumático, transtorno de estresse agudo, transtorno de ansiedade generalizada, transtorno de ansiedade devido a uma condição médica geral, transtorno de ansiedade induzido por substância, transtorno de ansiedade sem outra especificação. A terceira maior incidência é de transtornos de aprendizagem com 7,8\%, sendo que nessa categoria são incluídos o transtorno da leitura, transtorno de matemática, transtorno da expressão escrita e transtorno de aprendizagem sem outra especificação.

Tabela 4: Distribuição da amostra dos principais transtornos mentais e problemas mentais que acometem a população atendida nos serviços de saúde mental neste período.

\begin{tabular}{llcc}
\hline Dados pessoais & Variáveis & Freqüência & Porcentagem (\%) \\
\hline $\begin{array}{l}\text { Transtornos } \\
\text { Mentais }\end{array}$ & $\begin{array}{l}\text { Transtornos de déficit de } \\
\text { atenção e comportamento } \\
\text { dirruptivo }\end{array}$ & 162 & $28,7 \%$ \\
& Transtornos de Ansiedade & 54 & $9,6 \%$ \\
\hline $\begin{array}{l}\text { Transtornos de } \\
\text { Aprendizagem } \\
\text { Mentais }\end{array}$ & $\begin{array}{l}\text { Problemas nas relações } \\
\text { Condições Adicionais que } \\
\text { podem ser foco de } \\
\text { atenção clínica }\end{array}$ & 74 & $7,8 \%$ \\
& Agressividade & $15 \%$ & $13,3 \%$ \\
\end{tabular}


Um estudo feito em quatro escolas públicas brasileiras procurou constatar a prevalência de TDAH em crianças na fase escolar. Através dos estudos constatou-se que $13 \%$ das crianças tinham indicação de TDAH, uma prevalência mais elevada que a tradicionalmente mencionada $(3-5 \%)^{17}$. Na população atendida nos serviços de saúde mental infantil em Itajaí, constatou um índice de $28,7 \%$ dos casos, duas vezes maior do que o apontado no estudo citado acima, pelo fato de ter considerado todos os transtornos contidos nos transtornos de déficit de atenção e de comportamento disruptivo, constituindo-se, portanto uma amostra maior. Inferese também a estes altos índices de TDAH as desigualdades metodológicas presentes nos estudos, afinal enquanto o estudo supra citado visou uma determinada faixa etária (seis a doze anos de idade), o presente estudo visou outra (três a dez de idade); dentre os procedimentos de seleção aquele utilizou amostras populacionais, bem como visou o uso de diferentes fontes de informação, (professores, pais e "pacientes”). Todavia esta pesquisa enfocou uma população diferenciada (dados ambulatoriais e clínicos), utilizando como única fonte de informação os prontuários dos pacientes, os quais eram observados e categorizados de acordo com a queixa apresentada por aqueles que buscavam tais serviços. Desta forma, acredita-se que os diferentes tipos de delineamento de pesquisas relacionadas a este fenômeno esclareçam o porquê da brusca diferença encontrada na prevalência de $\mathrm{TDAH}^{18}$.

No que diz respeito aos sintomas de ansiedade, estima-se que quase $10 \%$ de todas as crianças e adolescentes preencherão critérios diagnósticos, em algum momento, para algum transtorno ansioso ${ }^{19}$. Os dados levantados no presente estudo são equivalentes a esses dados, demonstrando uma prevalência de 9,6\% de transtornos de ansiedade em crianças.

Conforme estudo realizado na Clínica Escola, na cidade de Itajaí, SC, constatou-se que os problemas de aprendizagem (32,99\%) re- presentavam a principal queixa apontada pelos informantes que acompanhavam as crianças que buscavam o serviço de psicologia, número mais elevado do que o constatado em nosso estudo que demonstrou um índice de (7,8\%) dos casos diagnosticados com transtorno de aprendizagem ${ }^{3}$. Mesmo com essa diminuição de casos, o transtorno de aprendizagem ainda aparece entre os três transtornos de saúde mental com maior incidência na população atendida nos serviços de saúde mental da cidade de Itajaí, SC.

A Tabela 4 apresenta dados relativos aos três problemas de comportamento com maior incidência encontrados na população atendida nos serviços de saúde mental. De acordo com os dados levantados no período de 2002 a 2007, foram constatados 18 diferentes problemas de comportamento, dentre eles com uma maior prevalência estão os problemas nas relações, com $13,3 \%$. Segundo o DSM-IV-TR, na categoria dos problemas nas relações, existe o problema de relacionamento que também é foco de atenção clínica associado a um transtorno mental ou condição médica geral, problema de relacionamento entre pai/mãe-criança, problema de relacionamento com o parceiro, problema de relacionamento com irmãos e problema de relacionamento sem outra especificação. De acordo com outra pesquisa realizada em clínica-escola, os problemas de relacionamento ou relacionais aparecem em 14,0\% dos casos de crianças atendidas, número bem semelhante ao apontado pelo presente estudo, de 13,3\% ${ }^{15}$.

Há alto índice de casos com problemas de relacionamento, advindos principalmente de famílias nucleares, ou seja, de constituição tradicional com pai, mãe e irmãos. Cabe a observação do número significativo de prontuários com informações desencontradas ou insuficientes, o que pode indicar dificuldades da família em fornecer tais dados.

Em seguida aparecem as condições adicionais que podem ser foco de atenção clínica com 2,7\%. De acordo com o DSM-IV-TR, es- 
tão incluídos nessa categoria os problemas de falta de aderência ao tratamento, simulação, comportamento anti-social em adulto, comportamento anti-social em criança ou adolescente, funcionamento intelectual borderline, declínio cognitivo relacionado à idade, luto, problema acadêmico, problema ocupacional, problema de identidade, problema religioso ou espiritual, problema de aculturação e problema de fase da vida. O terceiro maior problema de comportamento constatado foi à agressividade com 2,1\%.

Segundo os dados levantados dos 564 prontuários em 7,8\%, não constavam dados referentes a algum transtorno mental ou um problema de comportamento, esse índice deve-se pelo número de crianças que faltavam às sessões de avaliação, o que impedia o estabelecimento de hipóteses diagnósticas bem como pelo fato de algumas crianças serem trazidas pela família alegando alguma queixa que durante a avaliação não foi confirmada como transtorno mental ou outra condição clínica.

\section{REFERÊNCIAS}

1. Mandlhate C. Novos conhecimentos, novas esperanças. Entrevista concedida ao Programa de Saúde em África; 2002. (citado em 2002). Disponível em http:// www.afro.who.int/mentalhealth/ publications/ respostas_web_novas_esperanças.pdf $>$. (Acessado em 27/03/ 2007).

2. Guerra AMC. A psicanálise no campo da saúde mental infanto-juvenil. Psychë. 2005; 9 (15): 139-154.

3. Gevaerd EBG. Os pais e os motivos da procura do serviço de psicologia da UNIVALI - CCS [Trabalho de Conclusão de Curso]. Itajaí: Universidade do Vale do Itajaí; 2001.
A agressividade foi um problema com alta incidência apontado no estudo realizado na Clínica Escola, na cidade de Itajaí, SC, com $28,26 \%$ das queixas apresentadas pelos pais das crianças e ou adolescentes atendidos no ano $2000^{3}$. Todavia, na presente pesquisa identificou-se um número inferior quanto ao problema de agressividade com apenas $2,1 \%$ dos casos diagnosticados. No entanto, esse ainda aparece como um dos principais problemas apresentados pelas crianças e adolescentes atendidos nos serviços de saúde mental.

Essa pesquisa vem fortalecer a necessidade de uma maior preocupação no planejamento de políticas públicas para os problemas de saúde mental infanto-juvenil, é de extrema urgência devido à crescente demanda da população em questão para os serviços de saúde mental. Há também a necessidade de ações que busquem a prevenção e a promoção da saúde mental e uma atenção maior para os transtornos mentais já presentes nesta população para que possam ser tratados adequadamente.

4. Santos PL. Problemas de saúde mental de crianças e adolescentes atendidos em serviço público de psicologia infantil. Psicol estud. 2006; 2(11): 315-321.

5. Sanches NA. Estudo epidemiológico de clientes da clínica-escola do departamento de psicologia da universidade federal de Uberlândia [Dissertação de Mestrado]. Uberlândia: Pontifícia Universidade Católica; 1985.

6. Graminha SSV, Martins MAO. Estudo das características da população que procura o serviço de atendimento infantil no centro de psicologia aplicada da FFCLRP-USP. Psico.1993; 1(24): 119-130. 
7. Linhares MBM, Parreira VLC, Marturano AC, Sant'anna SC, et al. Caracterização dos motivos da procura de atendimento infantil em um serviço de psicopedagogia clínica. Medicina Ribeirão Preto. 1993; 2(26): 148-160.

8. Barbosa JI, Silvares EFM. Uma caracterização preliminar das clínicasescola de Fortaleza. Estud psicol. 1994; 11(3):50-6.

9. Borges SLP. Caracterização da clientela da clínica são marcos na área de atendimento infantil. Interações: estud pesqui psicol. 1996; 1(1):59-78.

10. Boarini ML, Borges, RF. Demanda infantil por serviços de saúde mental: sinal de crise. Estud psicol. 1998; 1(3): 83-108.

11. Bernardes-da-rosa LT, Garcia RM, Domingos, NAM, Silvares, EFM, et al. Caracterização do atendimento psicológico prestado por um serviço de psicologia a crianças com dificuldades escolares. Estud psicol. 2000; 3(17): 5-14.

12. Romaro RA, Capitao, CG. Caracterização da clientela da clínica escola de psicologia da universidade de são francisco. Psicol teor e prát. 2003; 1(5):111-121.

13. Henna ES, Abreu LC, Neto MLF, Reis AOA, et al. Rede de atenção à saúde mental de base comunitária: a experiência de Santo André. Rev bras crescimento desenvolv hum. 2008; 18 (1): 16-26.
14. Brasil. Ministério da Saúde, Secretaria de Atenção à Saúde, Departamento de Ações Programáticas Estratégicas. Marco teórico e referencial: saúde sexual e saúde reprodutiva de adolescentes e jovens. Brasília: Editora do Ministério da Saúde; 2006.

15. Melo SA, Perfeito HCS. Características da população infantil atendida em triagem no período de 2000 a 2002 numa clínica escola. Estud psicol. 2006; 3(23): 239-249.

16. American Psychiatric Association. Manual diagnóstico e estatístico de transtornos mentais. Porto Alegre: Artes Médicas; 2002.

17. Fontana RS, Vasconcelos MM, Werner J, Goes FV, Liberal EF, et al. Prevalência de tdah em quatro escolas públicas brasileiras. Arq neuropsiquiatr. 2006;1(65):134-7.

18. Rohde LA, Mattos P. Epidemiologia. In: Golfeto JH, Barbosa GA. Princípios e práticas em TDAH. Porto Alegre: Artes Médica; 2003. p. 15-34.

19. Asbahr FR.Transtornos ansiosos na infância e na adolescência. J pediatr. 2004; 80(2): 28-34.

Recebido em 14 de junho de 2009 Modificafo em 26 de setembro de 2009 A ceito em 13 de novembro de 2009 